\title{
Smart Air-Water Interfaces with Arylazopyrazole Surfactants and Their Role in Photoresponsive Aqueous Foam
}

\author{
Marco Schnurbus, ${ }^{\dagger}$ Lucas Stricker, ${ }^{\ddagger}$ Bart Jan Ravoo, ${ }^{\ddagger}{ }^{\dagger}$ and Björn Braunschweig ${ }^{* \dagger}{ }^{\dagger}$ \\ ${ }^{\dagger}$ Institute of Physical Chemistry and Center for Soft Nanoscience, Westfälische Wilhelms-Universität Münster, Corrensstraße 28/30, \\ 48149 Münster, Germany \\ ${ }^{\ddagger}$ Organic Chemistry Institute and Center for Soft Nanoscience, Westfälische Wilhelms-Universität Münster, Corrensstraße 40, 48149 \\ Münster, Germany
}

\section{Supporting Information}

ABSTRACT: A new light-switchable azo-surfactant arylazopyrazole tetraethylene glycol carboxylic acid $\left(\mathrm{AAP}-\mathrm{E}_{4}\right)$ was used as a molecular building block to functionalize macroscopic foams. AAP-E $E_{4}$ was studied in the bulk solution with $\mathrm{UV} /$ vis spectroscopy and at the interface with sum-frequency generation (SFG) as well as tensiometry. Additional foaming experiments were performed with a dynamic foam analyzer to study the role of $\mathrm{AAP}_{-} \mathrm{E}_{4}$ surfactants at the ubiquitous air-

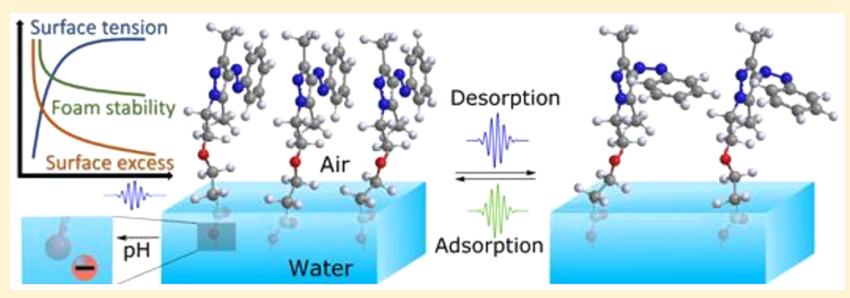
water interface as well as within macroscopic foam. In the bulk, it is possible to switch the AAP-E $\mathrm{E}_{4}$ surfactant reversibly from trans to cis configurations and vice versa using $380 \mathrm{~nm} \mathrm{UV}$ and $520 \mathrm{~nm}$ green light, respectively. At the interface, we demonstrate the excellent switching ability of AAP-E $\mathrm{E}_{4}$ surfactants and a substantial modification of the surface tension. In addition, we show that the response of the interface is strongly influenced by lateral electrostatic interactions, which can be tuned by the charging state of $\mathrm{AAP}-\mathrm{E}_{4}$. Consequently, the electrostatic disjoining pressure and thus the foam stability are highly dependent on the bulk $\mathrm{pH}$ and the charging state of the interface. For that reason, we have studied both the surface net charge (SFG) and the surface excess (tensiometry) as important parameters that determine foam stability in this system and show that neutral $\mathrm{pH}$ conditions lead to the optimal compromise between switching ability, surface excess, and surface charging. Measurements on the foam stability demonstrated that foams under irradiation with green light are more stable than foams irradiated with UV light.

\section{INTRODUCTION}

Foams find applications in many industrial processes or as materials from heat insulation to food colloids. ${ }^{1-3}$ Although foams are lightweight materials with unique properties such as their viscoelastic behavior, most foam properties cannot be changed after completion of the foaming process. Many applications demand high foam stability (FS), ${ }_{4}^{4}$ for example, for surface decontamination, but once this process is completed, it is preferable to switch the foam from stable to unstable conditions enabling easy removal and recycling of the foam constituents. Obviously, there is great interest to turn foams into materials that respond to external stimuli. Although the response of aqueous foam is required on macroscopic length scales, it originates from the ubiquitous air-water interfaces (IFs) as the major building blocks of aqueous foam. For that reason, smart air-water IFs, which are reconfigurable in situ, for various purposes are needed.

As a consequence, it is crucial to change the hydrophobicity and the intermolecular interactions of surface-active molecules with external parameters, such as light, ${ }^{5-9}$ temperature, ${ }^{1,8} \mathrm{pH}^{2}$ magnetic, or electric fields. ${ }^{10,11}$ The advantage of switching a surfactant with light is that it is easy to manipulate and that the stimulus can be localized both in time and space. ${ }^{12,13}$ Wellknown compounds that change their configurations upon irradiation are derivates of azobenzene, ${ }^{14,15}$ stilbene, ${ }^{16,17}$ and spiropyrane. ${ }^{18,19}$ Azobenzenes are particularly interesting because they switch between trans to cis configurations without any bond breaking. This change in the molecular configuration leads to a significant change in the electronic as well as the steric structure of the surfactant and thus allows for switching its surface activity.

For that reason, adaptive materials attract considerable interest in recent years and photoresponsive IF-controlled materials such as emulsions, ${ }^{20-22}$ organogels, ${ }^{23}$ vesicles, ${ }^{24}$ and microgels $^{25}$ have been reported. Previous works on lightswitchable foams have focused on azobenzene ionic ${ }^{5-8}$ and nonionic ${ }^{9}$ surfactants. Chevallier et al. ${ }^{6}$ studied amphiphilic azobenzene derivatives with a cationic head group and described the control of FS and the interfacial properties using light stimuli. In the latter work, the surface tension was higher by irradiation with UV light as compared to blue light conditions. In addition, Chevallier et al. show different foam stabilities in UV light as compared to foams produced in the dark. Lei et al. ${ }^{5}$ investigated azobenzene surfactants having an anionic head group and also show the switching ability of the surface tension as well of FS for different light conditions. After

Received: February 21, 2018

Revised: April 5, 2018

Published: May 2, 2018 
irradiation with UV light, the surface tension and the critical micelle concentration increased, whereas the FS decreased. Although previous works have clearly shown that foams and air-water IFs can be made responsive to light and other stimuli, to the best of our knowledge, both the switching kinetics and the reversibility of photoswitching processes at the air-water IF have not been reported so far.

In this article, we study the molecular structure, charging state, and switching kinetics of arylazopyrazole tetraethylene glycol carboxylic acid $\left(\mathrm{AAP}_{4} \mathrm{E}_{4}\right)$ (Figure 1 ) as a new light-

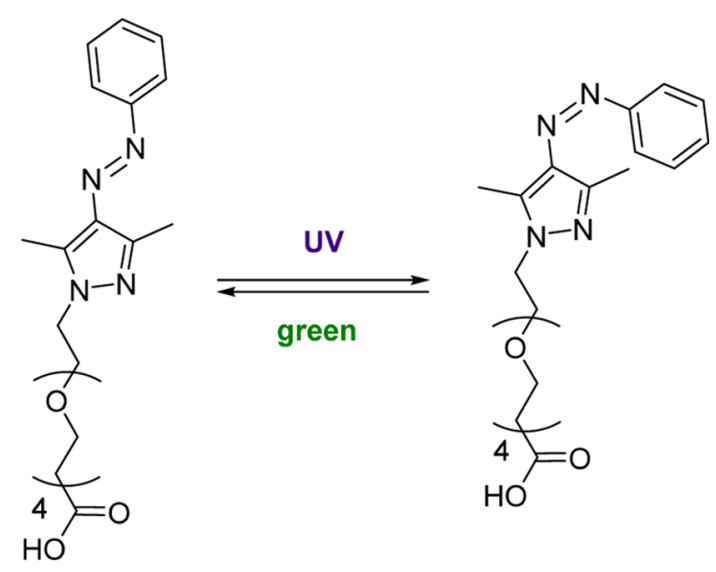

Figure 1. Molecular structure of $\mathrm{AAP}_{-} \mathrm{E}_{4}$ in both trans and cis conformation.

switchable surfactant for responsive air-water IFs. Arylazopyrazoles are superior analogues of azobenzenes with thermal halflife times superior compared to most azobenzenes and a smaller spectral overlap of the trans and cis isomers which results into a more favorable photostationary state with $>95 \%$ switching in both directions. ${ }^{26,27} \mathrm{AAP} \mathrm{E}_{4}$ surfactants show unprecedented reversibility of their interfacial molecular structures, which offer unique control on the IF tension and charging state of airwater IFs. To study the latter properties on a molecular level, we have applied both dynamic surface tension measurement and vibrational sum-frequency generation (SFG) and report on in situ measurements, which have been performed during the dynamic change of air-water IFs under light irradiation. In addition, we show the use of the AAP- $\mathrm{E}_{4}$ surfactant to stabilize macroscopic foam and study the effects of the interfacial molecular structure within macroscopic foam through structure-property relations.

\section{EXPERIMENTAL DETAILS}

Sample Preparation. The synthesis protocol for the arylazopyrazole surfactant is given in brief in the Supporting Information and explained in detail elsewhere. ${ }^{28}$ All chemicals used for the synthesis (see Supporting Information) of AAP-E ${ }_{4}$ surfactants were purchased from Alfa Aesar (Karlsruhe, Germany), Sigma-Aldrich Chemie (Taufkirchen, Germany), or TCI Europe (Zwijndrecht, Belgium) and used without further purification. Solvents were dried before use.

AAP- $E_{4}$ solutions were prepared by dissolving the azo-surfactant in ultrapure water $(18.2 \mathrm{M} \Omega \mathrm{cm}$; total oxidizable carbon $\leq 3 \mathrm{ppb})$, which was obtained from a Merck Milli-Q Reference A+ purification system.

The $\mathrm{pH}$ was adjusted for all samples (except for the lowest $\mathrm{pH}$ ) using $\mathrm{NaOH}$ (99.99\%, Alfa Aesar) dilutions and determined with a $\mathrm{pH}$ meter (FiveEasy20, Mettler Toledo). All necessary glassware was precleaned in Alconox solutions (Sigma-Aldrich), and after drying, all parts were soaked in concentrated sulfuric acid with NOCHROMIX (Godax Laboratories) for at least $12 \mathrm{~h}$. Subsequent to the latter treatment, all parts were thoroughly rinsed with ultrapure water. All samples were used immediately after preparation, and the measurements were performed at $295 \mathrm{~K}$ room temperature.

UV/Vis Spectroscopy. Ultraviolet/visible (UV/vis) absorption spectra were recorded with a PerkinElmer LAMBDA $650 \mathrm{UV} /$ vis spectrometer in the wavelength region of $250-700 \mathrm{~nm}$. The first spectrum was determined after the sample was irradiated with the green light-emitting diode (LED) for $5 \mathrm{~min}$. After that the sample was irradiated with the UV-LED for $5 \mathrm{~min}$ and another spectrum was measured. The same procedure was carried out for the green LED. Irradiation was carried out in four cycles, and the absorbance at the wavelength of $334 \mathrm{~nm}$ was recorded as a function of time, additionally.

Tensiometry. The surface tension was measured with a pendant drop tensiometer (DSA100, Krüss, Germany) using a modified cuvette. The cuvette was equipped with two long-pass filters (Schott OG590) with a cutoff at $590 \mathrm{~nm}$. This ensures that the light used to determine the drop shape did not affect the arylazopyrazole surfactant. For dynamic surface tension measurements, a drop was formed at the end of a syringe cannula and a charge-coupled device camera monitored its shape for different times after the drop was created. Using image analysis and the Young-Laplace equation, the surface tension was determined from the drop shape as a function of time until equilibrium was reached. ${ }^{29,30}$

Inside the cuvette, two LEDs with center wavelengths of 385 and $520 \mathrm{~nm}$ were placed and used for in situ photoswitching while the dynamic changes in surface tension were simultaneously recorded.

Vibrational SFG. SFG is a molecular spectroscopic method based on a second-order nonlinear optical effect. ${ }^{31,32}$ For SFG spectroscopy, we applied a home-built broadband spectrometer that is described elsewhere. ${ }^{33}$ At the IF, we overlapped a narrow-band time-asymmetric picosecond beam at $800 \mathrm{~nm}$ wavelength [full width at half-maximum (fwhm) bandwidth $<6 \mathrm{~cm}^{-1}$ ] with a tunable femtosecond infrared (IR) beam which has a broad bandwidth of $>200 \mathrm{~cm}^{-1} \mathrm{fwhm}$. In the overlap of both fundamental beams, a third beam was generated with the sum frequency $(\mathrm{SF}) \omega_{\mathrm{SF}}$ of the two fundamental frequencies. The intensity of the SF is in this second-order process dependent on the intensities of the two laser beams $\left(I_{\text {vis }}\right.$ and $\left.I_{\mathrm{IR}}\right)$ and on nonresonant $\chi_{\mathrm{NR}}^{(2)}$ and resonant parts $\chi_{\mathrm{R}}^{(2)}$ of the second-order nonlinear susceptibility $\chi^{(2)}$ as well as on a third-order contribution which is relevant for charged $\mathrm{IFs}^{34-36}$ with nonzero surface potentials $\psi_{0}$

$$
I\left(\omega_{\mathrm{SF}}\right) \propto\left|\chi_{\mathrm{NR}}^{(2)}+\chi_{\mathrm{R}}^{(2)}+\frac{\kappa}{\kappa+i \Delta k_{z}} \chi^{(3)} \psi_{0}\right|^{2} I_{\mathrm{IR}} I_{\mathrm{vis}}
$$

In this equation $\kappa$ is the inverse Debye length and $\Delta k_{z}$ is the wave vector mismatch between all beams. The resonant part

$$
\chi_{\mathrm{R}}^{(2)}=\sum_{k} \frac{A_{k}}{\omega_{k}-\omega_{\mathrm{IR}}+i \Gamma_{k}}
$$

is a function of the resonance frequency $\omega_{k}$ of a molecular vibration, the bandwidth $\Gamma_{k}$ of the vibrational mode $k$, and the oscillator strength $A_{k}=N a_{k} \mu_{k}$. Here, $A_{k}$ depends on $N$ the number density of the interfacial molecular species as well as on the orientational average of the Raman polarizability $a_{k}$ and the dynamic dipole moment $\mu_{k}$. The dependence on the orientational average of the SF amplitude has a farreaching consequence for the IF selectivity of the SFG method: for inversion symmetric systems such as bulk liquids and gases, this average is zero for symmetry reason, but IFs such as the air-water IF necessarily break the bulk symmetry and are thus the only source for SFG signals in such systems. For that reason, SFG is inherently IFspecific for an inversion symmetric system where only the few molecular layers at the IF can contribute.

The different contributions provide complementary information: $\chi_{\mathrm{R}}^{(2)}$ is highly specific for adsorbed molecules because tuning the IR pulse over molecular resonances at the IF leads to resonances in the SFG spectrum. In the case of charged IFs and small, highly polarizable interfacial molecules such as $\mathrm{H}_{2} \mathrm{O}$, the interfacial electric field induces additional polar order and polarization of the latter molecules at the IF. For that reason, the SF intensity of these molecules is also affected 
by the interfacial electric field. Because $\chi^{(3)}$ has the same frequency dependence as $\chi_{\mathrm{R}}^{(2)}$, the amplitude of a vibrational band in SFG spectra can be influenced by $\chi^{(3)}$ effects and is thus dependent on the surface potential which is directly coupled to the surface charge $\sigma_{0}$ by the Grahame equation.

$$
\sigma_{0}=\sqrt{8 c_{0} \varepsilon_{0} \varepsilon_{\mathrm{r}} k_{\mathrm{B}} T} \sinh \left(\frac{z e \psi_{0}}{2 k_{\mathrm{B}} T}\right)
$$

In our experiments, we have recorded SFG spectra in the range of $2800-3800 \mathrm{~cm}^{-1}$ by tuning the IR center frequency in seven steps. The acquisition time for each center frequency was $40 \mathrm{~s}$. For a reference, we used a plasma-cleaned polycrystalline gold surface and all sample spectra were normalized to this reference. In addition, we have also recorded in situ spectra while the surfactants where switched from cis to trans configuration. For these measurements, we tuned the IR center frequency again in seven steps but with an acquisition time of $10 \mathrm{~s}$ only.

Foam Characterization. For the foam characterization, a dynamic foam analyzer (DFA100, Krüss, Germany) was used to determine FS as a function of time. The foam analyzer was placed in an enclosed chamber to avoid unwanted irradiation, for example, from the room lights. The sample solution $(70 \mathrm{~mL})$ was filled in a glass column $(250$ $\mathrm{mm}$ length and a diameter of $40 \mathrm{~mm}$ ) with a porous glass frit (Carl Roth, Germany) which was fixed at the bottom of the column. A nitrogen gas with a flow rate of $0.15 \mathrm{~L} / \mathrm{min}$ was passed through the glass frit for $50-90 \mathrm{~s}$ to produce foams. With the DFA100, it is possible to measure the foam height as a function of foam age by measuring the light transmission through the glass column. For that purpose, an IR LED panel (which does not affect the switching of the surfactant) was installed at one side of the column and on the opposite side, a line sensor was installed. The FS was determined with FS $=\frac{H_{t}}{H_{0}} \cdot 100 \%$, where $H_{t}$ is the foam height at time $t$ and $H_{0}$ is the maximum foam height. Prior to foaming, we have irradiated the samples for $15 \mathrm{~min}$ with green/UV light inside the foam analyzer enclosure. This procedure initially avoids possible light scattering effects. During foaming and foam aging, irradiation was continued.

\section{RESULTS AND DISCUSSION}

UV/Vis Spectroscopy. Figure 2a presents the UV/vis absorption spectra of $0.1 \mathrm{mM} \mathrm{AAP-E_{4 }}$ surfactant dilutions at a solution $\mathrm{pH}$ of 7.1. As indicated in the figure, we compare the two spectra of solutions that were recorded after $30 \mathrm{~min}$ continuous irradiation with $520 \mathrm{~nm}$ green light and $380 \mathrm{~nm}$ UV light, respectively. Under green light conditions, we observed a strong absorption band with a maximum centered at the wavelength of $334 \mathrm{~nm}$. A much smaller band is centered at the wavelength of $420 \mathrm{~nm}$ and accompanies the band at $334 \mathrm{~nm}$. After exposing the sample with UV light, the absorbance at 334 $\mathrm{nm}$ decreased, whereas the absorbance due to a slightly shifted band at $420 \mathrm{~nm}$ increases. These substantial changes in the surfactant extinction spectra are indicative for photoisomerization of the arylazopyrazole surfactant from trans (green light) to cis (UV light) configuration (Figure 1).

In Figure $2 \mathrm{~b}$ we present the absorbance at $334 \mathrm{~nm}$ of $0.1 \mathrm{mM}$ AAP- $E_{4}$ for different cycles of irradiation using UV and green light. Close inspection of Figure $2 \mathrm{~b}$ shows that the switching process is highly reversible as we did not observe any changes in the peak absorbance between cycles. In Figure $2 \mathrm{c}$, the absorbance at $334 \mathrm{~nm}$ is shown as a function of time during which the sample solutions was irradiated with green or with UV light. To obtain quantitative information on the switching kinetics, we have additionally analyzed our UV/vis data in Figures $2 c$ and $S 1$ with respect to apparent rate constants $k$ for which we have assumed first-order kinetics of the light-induced conformational change from trans to cis and vice versa
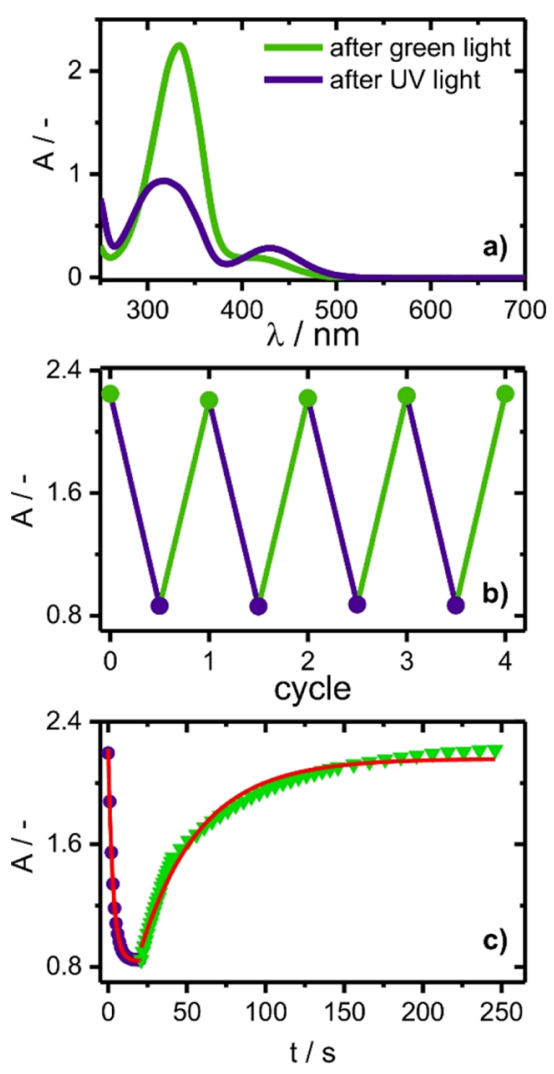

Figure 2. (a) UV/vis spectra of $0.1 \mathrm{mM} \mathrm{AAP}-\mathrm{E}_{4}$ surfactants in the aqueous solution ( $\mathrm{pH} 7.1$ ) after 5 min irradiation with $520 \mathrm{~nm}$ green light and $380 \mathrm{~nm}$ UV light as indicated in the figure. (b) Absorbance at $334 \mathrm{~nm}$ for four cycles of $5 \mathrm{~min}$ irradiation with UV and green light, respectively. (c) Absorbance at $334 \mathrm{~nm}$ wavelength as a function of irradiation time with UV (circles) and green light (triangles). The red solid lines in (c) represent fits to the experimental data according to eq 4 (main text).

$$
A(t)=A_{\text {eq }} \pm\left(A_{0} \mp A_{\text {eq }}\right) \mathrm{e}^{-k \cdot t}
$$

with $A_{\text {eq }}$ and $A_{0}$ being the absorbance in equilibrium after and before photoswitching, respectively. The photoisomerization was completed within $20 \mathrm{~s}$ after the UV light source was switched on. The kinetics of the reverse process (back switch) under green light conditions are much slower and require $\sim 230$ $\mathrm{s}$ until equilibrium is reached. In Table 1 , we present the rate

Table 1. Rate Constants $k$ for the Switching Kinetics from Trans To Cis Conformations in the Bulk and at the IF

\begin{tabular}{ccc} 
bulk & $\mathrm{pH} 4.4$ & $\mathrm{pH} \mathrm{7.1}$ \\
$k_{\text {trans } \rightarrow \text { cis }}^{\text {bulk }} / \mathrm{min}^{-1}$ & 20.6 & 20.3 \\
$k_{\text {cis } \rightarrow \text { trans }}^{\text {bulk }} / \mathrm{min}^{-1}$ & 1.7 & 1.6 \\
IF & $\mathrm{pH} 3.9$ & $\mathrm{pH} 6.9$ \\
$k_{\text {trans } \rightarrow \text { cis }}^{\mathrm{IF}} / \mathrm{min}^{-1}$ & 1.0 & 0.7 \\
$k_{\text {cis } \rightarrow \text { trans }}^{\mathrm{IF}} / \mathrm{min}^{-1}$ & 0.2 & 0.1 \\
\hline
\end{tabular}

constants $k^{\text {bulk }}$ for $\mathrm{pH} 4.4$ and 7.1. Obviously, the conformational change from trans to cis in the bulk $\left(k_{\text {trans } \rightarrow \text { cis }}^{\text {bulk }}\right)$ is by a factor 12 faster as the change from cis to trans $\left(k_{\text {cis } \rightarrow \text { trans }}^{\text {bul }}\right)$. At this point, it is now interesting to compare the bulk behavior for different $\mathrm{pH}$ values as we will also address interfacial properties at different $\mathrm{pH}$ values below. Using Table 1, we can directly compare the apparent rate constant for $\mathrm{pH} 4.4$ and 7.1, 
which are within the limits of the experimental error identical. This observation is consistent with steady-state UV/vis spectra at different $\mathrm{pH}$ values, which are independent of $\mathrm{pH}$ as well (Figure S1).

Surface Tension Measurements. Increasing the $\mathrm{pH}$ value causes deprotonation of the AAP- $\mathrm{E}_{4}$ surfactant carboxylic acid group and consequently leads to a more negatively charged air-water IF. To investigate at which $\mathrm{pH}$ conditions the maximum in surface charging is reached, we have recorded both the surface tension and vibrational SFG spectra for different bulk $\mathrm{pH}$ values. In Figure 3a, we present the equilibrium surface

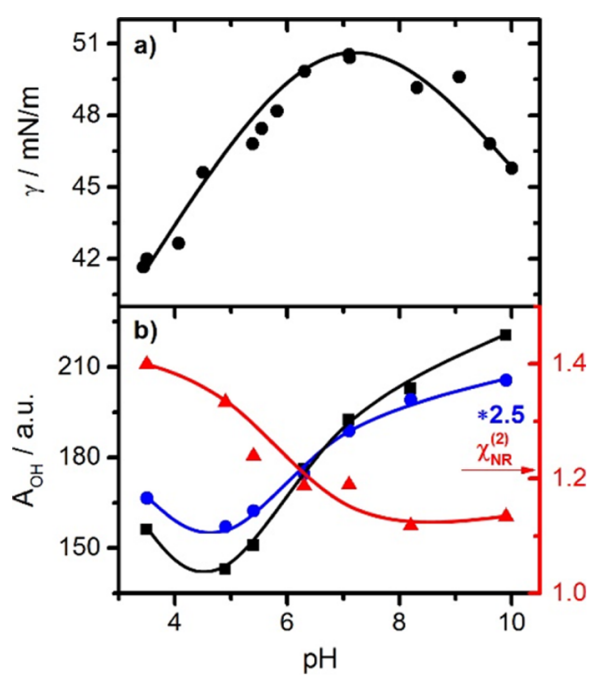

Figure 3. (a) $\mathrm{pH}$ dependence of the equilibrium surface tension $\gamma$ of air-water IFs that were modified by $1 \mathrm{mM} \mathrm{AAP}-\mathrm{E}_{4}$ surfactants. We point out that the ionic strength is changing with the solution $\mathrm{pH}$. Arguments why we have not added a background electrolyte to keep the ionic strength constant are presented in the General Discussion section. (b) SFG amplitudes $A_{\mathrm{OH}}$ of $\mathrm{O}-\mathrm{H}$ stretching bands from interfacial water molecules at $3450 \mathrm{~cm}^{-1}$ (black squares) and 3200 $\mathrm{cm}^{-1}$ (blue circles; multiplied by a factor of 2.5 ) as well as the nonresonant $\chi_{\mathrm{NR}}^{(2)}$ contribution (red triangles) from SFG spectra are shown in Figure 5a and in the Supporting Information. The concentration of AAP-E $\mathrm{E}_{4}$ surfactants was $1 \mathrm{mM}$ for all samples.

tension as a function of the bulk $\mathrm{pH}$. Increasing the $\mathrm{pH}$ from 4 to 7 leads to an increase in surface tension from $41.7 \mathrm{mN} / \mathrm{m}$ to a local maximum in surface tension of $50.1 \mathrm{mN} / \mathrm{m}$. Note that these experiments were done at a different ionic strength $[\mathrm{pH} 4$ $(0 \mathrm{mM}), 7(6.4 \mathrm{mM}), 9(11.4 \mathrm{mM})]$, and we point out that both the choice of the counter ion (here $\mathrm{Na}^{+}$and $\mathrm{H}_{3} \mathrm{O}^{+}$) as well as the overall ionic strength will affect the results in Figure 3. However, addressing ion-specific effects is beyond the scope of this study; we have particularly chosen the lowest possible ionic strength for each $\mathrm{pH}$ value because this is relevant for foam stabilization as we will discuss in the General Discussion. Besides the equilibrium values of the surfactant IF tension, nonequilibrium surface tensions are important to address the interfacial adsorption and switching kinetics of the AAP- $E_{4}$ surfactant.

The latter are both presented in Figure 4. Here, we compare the dynamic surface tension for the surfactant adsorption with and without switching the surfactant from trans to cis configuration. In both cases, air-water IFs were generated, and the surface tension was monitored over time. After $1800 \mathrm{~s}$, the irradiation was changed from green to UV light, which
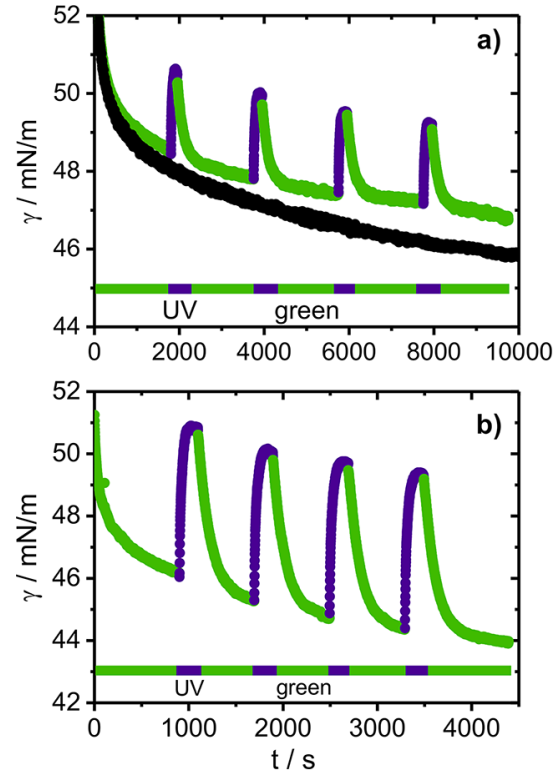

Figure 4. (a,b) dynamic surface tensions $\gamma(t)$ during adsorption and

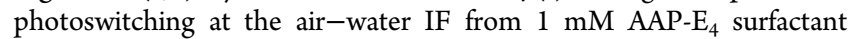
solutions for bulk $\mathrm{pH}$ values of 6.9 and 3.9. Data points in green and purple indicate surface tension changes during green and UV light irradiation, respectively. Data points in black color show a reference measurement without photoswitching of the AAP- $\mathrm{E}_{4}$ surfactant. Note that the curves do not overlap because of the superimposed adsorption kinetics.

caused a subsequent fast and substantial increase in surface tension. Equilibrium is observed $200 \mathrm{~s}$ after the UV light was turned on. At this point, the UV light was turned off, while the green light was turned on and caused a decrease of the surface tension. However, the time until equilibrium was reached is in this case was longer and is indicative for slower kinetics for the back-switch. This procedure was repeated in four subsequent cycles, which are also shown in Figure 4. We conclude from a close comparison of the changes in surface tension in Figure 4 that the switching of the surface tension at the air-water IF is highly reversible and is caused by the photoisomerization of the surfactant as explained above.

We point out that the differences between the dynamic surface tension with and without light switching are associated with the superimposed adsorption kinetics of the surfactants. The latter are much slower compared to the light-induced changes, and thus, a quantitative comparison of the surface tensions should not be made. We have also studied the photoswitching of the surface tension for different $\mathrm{pH}$ values. Although, the reversibility of the interfacial molecular switch (compare Figures $4 \mathrm{a}$ vs $4 \mathrm{~b}$ and S3) and its bulk behavior (Figures 2 and S1) are independent of $\mathrm{pH}$, the switching ability of the IF in terms of changes in the equilibrium surface tension $\Delta \gamma$ under different light irradiations did change with the bulk $\mathrm{pH}$ and was higher for acidic compared to $\mathrm{pH}$ neutral or basic conditions (Figure S3). Partly, this behavior is caused by a lower surface excess at neutral and basic $\mathrm{pH}$ values as we will discuss below.

To get quantitative information on the adsorption/ desorption rates, we have modeled the dynamic changes in surface tension (Figure 4) using a Gibbs-Langmuir isotherm as a simple model for the adsorption process. This model is chosen because it offers the fewest possible unknown 


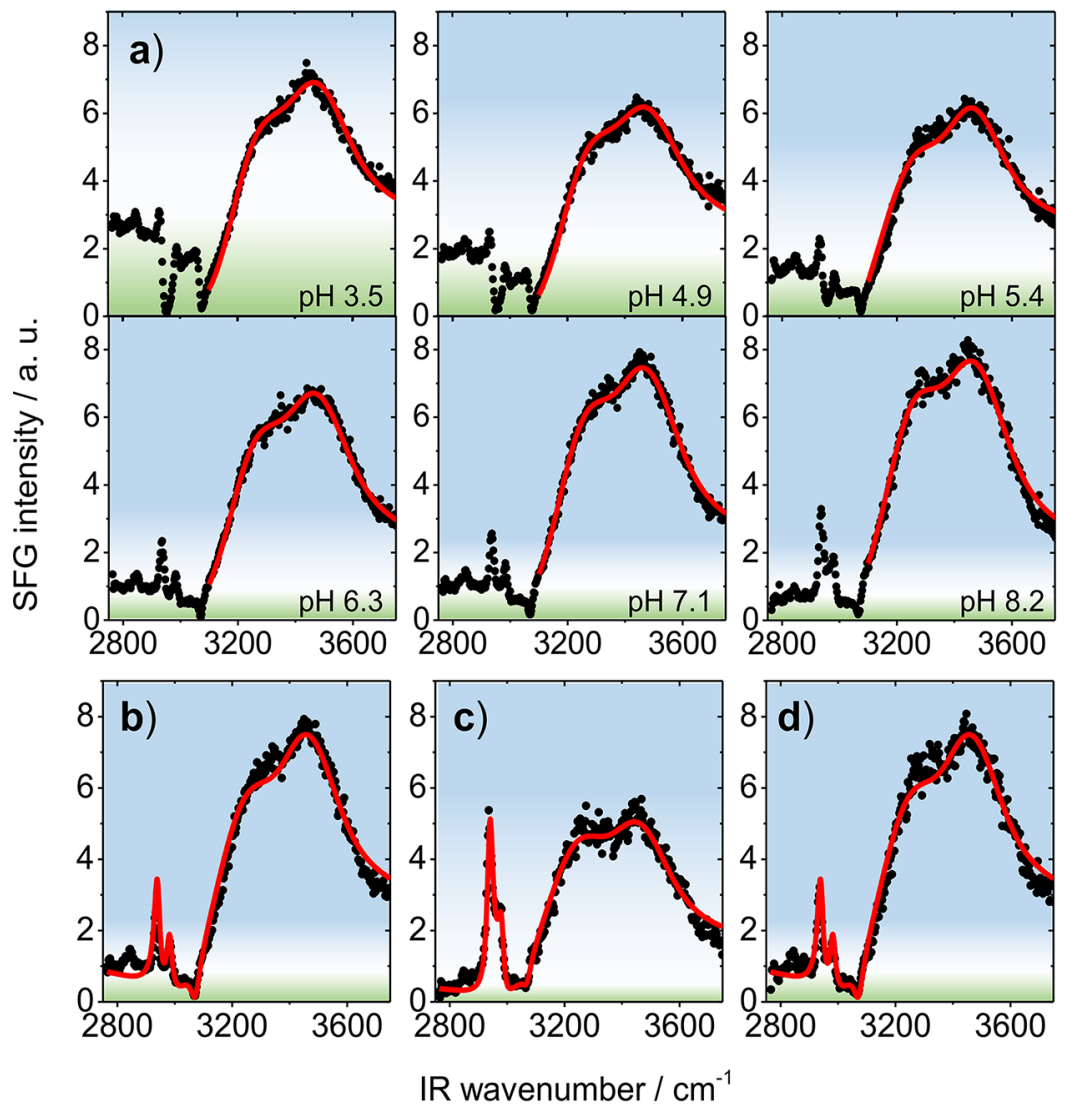

Figure 5. (a) Vibrational SFG spectra of air-water IFs from $1 \mathrm{mM} \mathrm{AAP-E_{4 }}$ solutions with $\mathrm{pH}$ values as indicated in the figure. SFG spectra of irradiated air-water IFs with $1 \mathrm{mM} \mathrm{AAP-E_{4 }}$ at $\mathrm{pH} 7.1$ and (b) control experiment before photoswitching, (c) after $1200 \mathrm{~s}$ irradiation with $380 \mathrm{~nm}$ UV light, and (d) after $1200 \mathrm{~s}$ irradiation with green light subsequent to (c). The solid red lines represent nonlinear least square fits to the experimental data as explained in the Vibrational SFG details section. In (d), the fit to the data in (b) is shown for comparison. The blue and green background colors reflect qualitatively the strength of nonresonant and resonant $\mathrm{O}-\mathrm{H}$ contributions to the spectra. For a quantitative comparison, the reader is referred to Figure $3 b$.

parameters in our fitting procedures and provides excellent fitting results, as shown in the Supporting Information (Figures S4 and S5). According to the Szyskowski's equation, we can write for the change in surface tension

$$
\gamma(t)=\gamma_{0} \pm R T \Gamma_{0} \ln \left(1-\frac{\Gamma(t)}{\Gamma_{0}}\right)
$$

with $\gamma_{0}$ and $\Gamma_{0}$ as well as $\gamma(t)$ and

$$
\Gamma(t)=\Gamma_{0} \cdot \mathrm{e}^{-k^{\mathrm{IF}} t}
$$

being the surface tension and the surface excess before and during photoswitching with the apparent rate constant $k^{\mathrm{IF}}$ for adsorption or desorption from the air-water IF. For this model, we assume that the conformational change is much faster than the adsorption/desorption kinetics and thus can be neglected compared to the latter. Note that we do not distinguish between two ionic states of AAP- $\mathrm{E}_{4}$ like Boyer et al. as well as Badban and co-workers did for other carboxylic acid surfactants. ${ }^{37,38}$ For that reason, we report in our study an apparent rate constant, which describes the combined action of both species. The reason why we resort to a simplified model is that it reduces the number of free parameters for fitting the experimental results and enables a comparison of the (overall) kinetics during photoswitching as a function of $\mathrm{pH}$ as well as between the IF and the bulk (see below).
In Table 1, we present the results for the rate constants for two different $\mathrm{pH}$ values. Comparing the apparent rate constant at $\mathrm{pH} 3.9$ with the rate constant at $\mathrm{pH} 6.9$, we observe a $30 \%$ decrease in the rate constant for the photoisomerization from trans to cis, whereas the rate constant from cis to trans decreases by $50 \%$. In addition, a comparison of the absolute values for the rate constants in the bulk with those at the IF shows 20 -fold faster kinetics in the bulk. This corroborates our earlier assumption that the kinetics of the photoisomerization can be neglected compared to the interfacial adsorption/ desorption kinetics. As we will discuss below, the origin of the different interfacial kinetics is due to the different charging state of the adsorbed AAP-E $\mathrm{E}_{4}$ surfactants at the air-water IF.

Vibrational SFG Spectroscopy. Figure 5a shows the vibrational SFG spectra that were recorded for $1 \mathrm{mM}$ arylazopyrazole (AAP-E - $_{4}$-modified air-water IFs at different bulk $\mathrm{pH}$ values. The SFG spectra in Figure 5 are dominated by broad bands between 3100 and $3800 \mathrm{~cm}^{-1}$, which can be attributed to $\mathrm{O}-\mathrm{H}$ stretching vibrations of interfacial water molecules. Two additional but narrow vibrational bands are centered at 2930 and $2980 \mathrm{~cm}^{-1}$ and can be attributed to the $\mathrm{CH}_{3}$ Fermi resonance and $\mathrm{CH}_{3}$ antisymmetric stretching vibrations of interfacial arylazopyrazole surfactants, respectively. A much weaker band at $3060 \mathrm{~cm}^{-1}$ is due to aromatic $\mathrm{C}-\mathrm{H}$ stretching vibrations and is also caused by interfacial arylazopyrazole moieties. All vibrational bands in Figure 5 show dispersive line shapes which are caused by the 
interference with a strong nonresonant contribution $\chi_{\mathrm{NR}}^{(2)} \cdot \chi_{\mathrm{NR}}^{(2)}$ is also due to the presence of interfacial arylazopyrazole surfactants. Obviously, there are no clear signatures for symmetric methyl stretching vibrations at $2878 \mathrm{~cm}^{-1}$ discernable in our SFG spectra, and only weak contributions from symmetric methylene stretching vibrations at $2850 \mathrm{~cm}^{-1}$ have been observed. The absence of symmetric methyl stretching vibrations is not surprising and is a cause of the molecular structure of the arylazopyrazole surfactant, which has local inversion symmetry at the pyrazole center. The weakness of symmetric methylene stretching vibrations from the tetraethylene glycol $\mathrm{E}_{4}$ chains is indicative to a significant number of Gauche conformations within the chain which lead to molecular structures with local inversion symmetry and thus negligible SFG signals. This conclusion is corroborated by previous Neutron scattering experiments at air-water IFs that were modified by $\mathrm{E}_{6}$ ethylene glycol nonionic surfactants. ${ }^{39}$

We performed nonlinear least square fits to the $\mathrm{pH}$ dependent SFG spectra of AAP-E - $_{4}$-modified air-water IFs in Figures $5 \mathrm{a}$ and $\mathrm{S} 7$. For that we have assumed model functions for the resonant-effective second-order susceptibility, which we assume as the sum of second-order and third-order contributions (see Vibrational SFG details section). Figure $3 \mathrm{~b}$ summarizes our fitting results. Here, we present the $\mathrm{pH}$ dependence of $\mathrm{O}-\mathrm{H}$ amplitudes from both the low- and highfrequency bands at 3200 and $3450 \mathrm{~cm}^{-1}$, respectively, as well as the $\mathrm{pH}$ dependence of the nonresonant contribution $\chi_{\mathrm{NR}}^{(2)}$. With increasing $\mathrm{pH}$ value, the latter decreased in amplitude, which was accompanied by an increase in $\mathrm{O}-\mathrm{H}$ amplitudes to the $\mathrm{SFG}$ spectra. Because the change in $\chi_{\mathrm{NR}}^{(2)}$ is directly related to the surface excess of AAP- $\mathrm{E}_{4}$ surfactants, ${ }^{40}$ we can conclude that the latter decreased with increasing $\mathrm{pH}$. This is consistent with the increase in surface tension as we have discussed above (Figure 3a).

The decrease in surface excess with the solution $\mathrm{pH}$ is associated by an increase in surface charging, which we infer from the increase in $\mathrm{O}-\mathrm{H}$ amplitudes.

In Figure 5b, we show the change in SFG spectra while the air-water IF with arylazopyrazole surfactant was irradiated with UV light for $1200 \mathrm{~s}$ until equilibrium was reached. Subsequently, the samples were subjected to green light for another $1200 \mathrm{~s}$ and a SFG spectrum of the IF was recorded once the IF was in equilibrium. From a close inspection of Figure $5 \mathrm{~b}$, it becomes obvious that both the $\mathrm{O}-\mathrm{H}$ intensity and the nonresonant contribution decreased after the sample was irradiated with UV light. The spectrum after subsequent irradiation with green light is within the experimental scatter identical to the spectrum before UV irradiation. This clearly demonstrates the high reversibility of the photoisomerization of the AAP-E 4 surfactant at air-water IFs. However, from the above observations, we can also conclude that the surfactants desorb and readsorb to the IF and thus reversibly modulate both the surface charge and the surface excess at the air-water IF as a function of light irradiation and wavelength.

Foaming Experiments. To test our conclusions on the interfacial charging state as a function of $\mathrm{pH}$ and light conditions, we have performed complementary experiments on the stability of macroscopic foams stabilized by AAP- $\mathrm{E}_{4}$ surfactants. The FS was measured for different $\mathrm{pH}$ values under UV light conditions, see Figure 6a, where the foam height was recorded as a function of time after foaming was completed.

The foam height changes in Figure $6 a$, clearly show that increasing the $\mathrm{pH}$ value leads to more stable foams with a

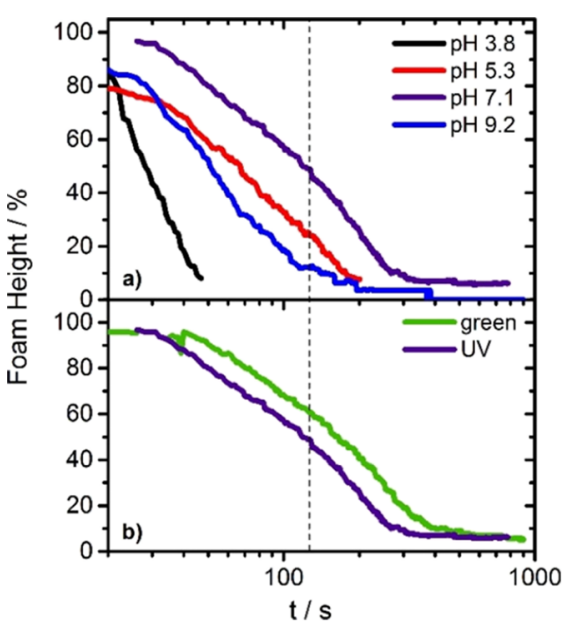

Figure 6. (a) Foam height as a function of time for different $\mathrm{pH}$ values under UV light conditions. (b) FS at pH 7.1 after the sample was irradiated with green light and with UV light. The vertical dashed lines represent half lifetime of the foam from $\mathrm{pH} 7.1$ solutions. All foams were stabilized with $1 \mathrm{mM} \mathrm{AAP-E_{4 }}$ surfactants.

longer lifetime. Foams from $\mathrm{pH} 7$ solutions showed the highest lifetime, whereas a further increase in solution $\mathrm{pH}$ leads to a decrease in FS. A comparison of foam stabilities under green light conditions shows comparable changes with $\mathrm{pH}$ (Supporting Information). Figure $6 \mathrm{~b}$ compares the stability at $\mathrm{pH} 7.1$ for green and UV light irradiation. Obviously, the foam that is irradiated with green light showed a much higher stability. At other $\mathrm{pH}$ values, we observe the same behavior, but the responsiveness of the foam decreased with increasing $\mathrm{pH}$ (see Supporting Information). These results can be compared with the surface tension measurements.

General Discussion. Our UV/vis measurements demon-

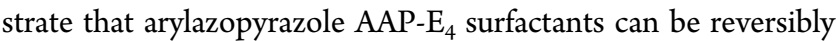
photoisomerized from trans to cis configuration. At acidic conditions, the surfactants are less charged but become increasingly charged with increasing $\mathrm{pH}$. SFG spectra and $\mathrm{O}-\mathrm{H}$ amplitudes from air-water IFs indicate that the surface charging of $\mathrm{AAP}-\mathrm{E}_{4}$-modified air-water interfaces saturates at near to neutral $\mathrm{pH}$ conditions. At these conditions, also the surface tension has a local maximum, which is due to a lower surface excess of the arylazopyrazole surfactants. This decrease in surface excess, which is caused by an increase in repulsive electrostatic interactions at the IF, is consistent with the increase in surface tension and the decrease in the nonresonant

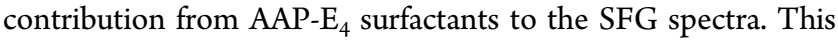
increase in electrostatic interactions causes a decrease in the apparent Gibbs free energy of adsorption $\Delta G_{\text {eff }}$ and thus a decrease in the apparent equilibrium constant. The surface excess $\Gamma$, as determined from the Gibbs adsorption isotherms at $\mathrm{pH} 3.8$ and 6.9 in Figure S6, is found to be $3.5 \mu \mathrm{mol} / \mathrm{m}^{2}(\sim 2$ molecules $\left./ \mathrm{nm}^{2}\right)$ and $2.5 \mu \mathrm{mol} / \mathrm{m}^{2}\left(\sim 1.5\right.$ molecules $\left./ \mathrm{nm}^{2}\right)$ for $\mathrm{pH} 3.8$ and 6.9, respectively. These differences bring strong support to our earlier conclusions of lower apparent equilibrium constants at higher $\mathrm{pH}$. As a consequence, the rate constant for IF adsorption is also decreased. This is corroborated by the differences in IF switching kinetics at $\mathrm{pH}$ 3.8 and 6.9 (Table 1). Our data show that the IF with low or negligible net charge at $\mathrm{pH} 3.8$ shows faster switching kinetics as compared to the fully charged IF at $\mathrm{pH} 6.9$ as evidenced by our SFG results (Figures $3 \mathrm{~b}$ and 5 ). Once all surfactant 
molecules at the IF are deprotonated, the interfacial electric field cannot be increased further and an additional increase in solution $\mathrm{pH}$ only leads to higher ionic strengths and thus higher screening of the interfacial electric field.

The FS in Figure 6 shows a strong dependence on the interfacial charging state. Obviously, the major stabilization mechanism of the foam with the AAP- $\mathrm{E}_{4}$ surfactants is via the electrostatic disjoining pressure. For that reason, it is not surprising that the highest stability is observed around $\mathrm{pH} 7$. Although at this $\mathrm{pH}$, the surface excess is lower, it provides the maximum surface charging and thus the maximum repulsion forces inside foam lamella. Note that there are other possible foam (de)stabilization mechanisms such as the surface rheology of the surfactant, ${ }^{41}$ Ostwald ripening, coalescence, steric interactions, and drainage. ${ }^{42}$ Other factors, such as a different velocity of the surfactant layer and the liquid flow underneath, reported previously by Blanc et al. ${ }^{43}$ as well as the formation of "caps" in the study on foam films by Mamane et al. ${ }^{7}$ could also play a role but are beyond the scope of our study while the formation of weak hydrogen bonds between neutral carboxylic acid groups with interfacial water molecules can be ruled out. This is because hydrogen bonding is likely to be stronger between neutral carboxylic acid head groups at a $\mathrm{pH}$ close to the head group $\mathrm{p} K_{\mathrm{a}}{ }^{44,45}$ Because the foams presented in our study are less stable at $\mathrm{pH} 3.8$ - where foam stabilization due to specific hydrogen bonding could play a role-we can conclude that hydrogen bonding is in our case not a relevant factor for the stability AAP-E $\mathrm{E}_{4}$ foams (Figure 6).

Once we reduce the electrostatic repulsion forces, for example, by decreasing the $\mathrm{pH}$ or by increasing the ionic strength, the FS is substantially reduced. Note that with increasing the $\mathrm{pH}$, the ionic strength also increases, which is the reason why we have not introduced an additional background electrolyte to keep the ionic strength with increasing bulk $\mathrm{pH}$ constant. Photoswitching of the surfactants from trans to cis configuration at the air-water IF shows that cis isomers at the IF lead to a lower surface excess and surface charging the electrostatic disjoining pressure is reduced and the foam becomes less stable.

\section{CONCLUSIONS}

We studied the ability of AAP- $\mathrm{E}_{4}$ to modify the surface tension and the charging state of air-water IFs as a function of external light stimuli using dynamic surface tension measurements and vibrational SFG spectroscopy.

The unique surfactant switching ability and the switching reversibility in the bulk and at the IF was shown as a function of $\mathrm{pH}$ and for irradiation with $380 \mathrm{~nm} \mathrm{UV}$ and $520 \mathrm{~nm}$ green light. Results on the behavior of AAP- $\mathrm{E}_{4}$ surfactants at the airwater IF demonstrate that irradiation with UV light causes desorption of molecules from the IF and thus a reduction in surface charging. Green light irradiation changed both the surface tension as well as the surface charging state reversibly back to their initial values before UV light irradiation. Bulk solutions with a $\mathrm{pH}$ of $\sim 7$ provide the IFs with the highest surface charging, whereas the interfacial electric double layer is only weakly screened by the electrolyte ionic strength.

In addition, we propose that the foams are electrostatically stabilized and thus show the highest FS at $\mathrm{pH} 7$ and a substantial reduction in stability under UV light conditions. This responsiveness of aqueous foam from AAP- $\mathrm{E}_{4}$ dilutions is a direct consequence of their switching ability at air-water IFs. Because the latter are a major building block of aqueous foam, controlling the IF with light-switchable molecular building blocks such as AAP-E $E_{4}$ directly leads to molecular control of macroscopic foam properties via smart air-water IFs.

\section{ASSOCIATED CONTENT}

\section{S Supporting Information}

The Supporting Information is available free of charge on the ACS Publications website at DOI: 10.1021/acs.langmuir.8b00587.

$\mathrm{UV} /$ vis measurements for $\mathrm{pH} 7.1$ and fitting of the kinetic curves; tensiometry data for different $\mathrm{pH}$ values and concentrations; fitting of the tensiometry data; SFG and foam measurements for different $\mathrm{pH}$ values; and the synthesis of the AAP-E $\mathrm{E}_{4}$ surfactant (PDF)

\section{AUTHOR INFORMATION}

\section{Corresponding Author}

*E-mail: braunschweig@uni-muenster.de.

ORCID $\odot$

Bart Jan Ravoo: 0000-0003-2202-7485

Björn Braunschweig: 0000-0002-6539-1693

Notes

The authors declare no competing financial interest.

\section{ACKNOWLEDGMENTS}

The authors are grateful for the support by Professor Dr. Ing. Wolfgang Peukert (FAU Erlangen-Nürnberg) and the funding from the European Research Council (ERC) under the European Union's Horizon 2020 research and innovation program (grant agreement no 638278). This work was in part funded by the Volkswagen Foundation.

\section{REFERENCES}

(1) Fameau, A.-L.; Saint-Jalmes, A.; Cousin, F.; Houssou, B. H.; Novales, B.; Navailles, L.; Nallet, F.; Gaillard, C.; Boué, F.; Douliez, J.P. Smart Foams: Switching Reversibly between Ultrastable and Unstable Foams. Angew. Chem. 2011, 123, 8414-8419.

(2) Middelberg, A. P. J.; Dimitrijev-Dwyer, M. A designed biosurfactant protein for switchable foam control. ChemPhysChem 2011, 12, 1426-1429.

(3) Shi, S.; Yin, T.; Shen, W. Switchable foam control by a new surface-active ionic liquid. RSC Adv. 2016, 6, 93621-93625.

(4) Carl, A.; von Klitzing, R. Smart foams: new perspectives towards responsive composite materials. Angew. Chem. 2011, 50, 1129011292.

(5) Lei, L.; Xie, D.; Song, B.; Jiang, J.; Pei, X.; Cui, Z. Photoresponsive Foams Generated by a Rigid Surfactant Derived from Dehydroabietic Acid. Langmuir 2017, 33, 7908-7916.

(6) Chevallier, E.; Monteux, C.; Lequeux, F.; Tribet, C. Photofoams: remote control of foam destabilization by exposure to light using an azobenzene surfactant. Langmuir 2012, 28, 2308-2312.

(7) Mamane, A.; Chevallier, E.; Olanier, L.; Lequeux, F.; Monteux, C. Optical control of surface forces and instabilities in foam films using photosurfactants. Soft Matter 2017, 13, 1299-1305.

(8) Salonen, A.; Langevin, D.; Perrin, P. Light and temperature biresponsive emulsion foams. Soft Matter 2010, 6, 5308.

(9) Chen, S.; Wang, C.; Yin, Y.; Chen, K. Synthesis of photoresponsive azobenzene molecules with different hydrophobic chain length for controlling foam stability. RSC Adv. 2016, 6, 60138-60144.

(10) Brown, P.; Bushmelev, A.; Butts, C. P.; Cheng, J.; Eastoe, J.; Grillo, I.; Heenan, R. K.; Schmidt, A. M. Magnetic Control over Liquid Surface Properties with Responsive Surfactants. Angew. Chem. 2012, 124, 2464-2466. 
(11) Rosslee, C.; Abbott, N. L. Active control of interfacial properties. Curr. Opin. Colloid Interface Sci. 2000, 5, 81-87.

(12) Cicciarelli, B. A.; Hatton, T. A.; Smith, K. A. Dynamic surface tension behavior in a photoresponsive surfactant system. Langmuir 2007, 23, 4753-4764.

(13) Schumers, J.-M.; Fustin, C.-A.; Gohy, J.-F. Light-responsive block copolymers. Macromol. Rapid Commun. 2010, 31, 1588-1607.

(14) Bandara, H. M. D.; Burdette, S. C. Photoisomerization in different classes of azobenzene. Chem. Soc. Rev. 2012, 41, 1809-1825.

(15) Orihara, Y.; Matsumura, A.; Saito, Y.; Ogawa, N.; Saji, T.; Yamaguchi, A.; Sakai, H.; Abe, M. Reversible Release Control of an Oily Substance Using Photoresponsive Micelles. Langmuir 2001, 17, 6072-6076.

(16) Takahashi, Y.; Kishimoto, M.; Kondo, Y. Photoinduced formation of threadlike micelles from mixtures of a cationic surfactant and a stilbene amphiphile. J. Colloid Interface Sci. 2016, 470, 250-256.

(17) Eastoe, J.; Dominguez, M. S.; Wyatt, P.; Beeby, A.; Heenan, R. K. Properties of a Stilbene-Containing Gemini Photosurfactant: LightTriggered Changes in Surface Tension and Aggregation. Langmuir 2002, 18, 7837-7844.

(18) Shi, Z.; Peng, P.; Strohecker, D.; Liao, Y. Long-lived photoacid based upon a photochromic reaction. J. Am. Chem. Soc. 2011, 133, 14699-14703.

(19) Halbritter, T.; Kaiser, C.; Wachtveitl, J.; Heckel, A. PyridineSpiropyran Derivative as a Persistent, Reversible Photoacid in Water. J. Org. Chem. 2017, 82, 8040-8047.

(20) Eastoe, J.; Dominguez, M. S.; Cumber, H.; Wyatt, P.; Heenan, R. K. Light-Sensitive Microemulsions. Langmuir 2004, 20, 1120-1125.

(21) Eastoe, J.; Sanchez-Dominguez, M.; Cumber, H.; Burnett, G.; Wyatt, P.; Heenan, R. K. Photoresponsive Microemulsions. Langmuir 2003, 19, 6579-6581.

(22) Takahashi, Y.; Fukuyasu, K.; Horiuchi, T.; Kondo, Y.; Stroeve, P. Photoinduced demulsification of emulsions using a photoresponsive gemini surfactant. Langmuir 2014, 30, 41-47.

(23) Zhou, Y.; Xu, M.; Wu, J.; Yi, T.; Han, J.; Xiao, S.; Li, F.; Huang, C. A novel photo-responsive organogel based on azobenzene. J. Phys. Org. Chem. 2008, 21, 338-343.

(24) Eastoe, J.; Vesperinas, A.; Donnewirth, A.-C.; Wyatt, P.; Grillo, I.; Heenan, R. K.; Davis, S. Photodestructible vesicles. Langmuir 2006, 22, 851-853.

(25) Bradley, M.; Vincent, B.; Warren, N.; Eastoe, J.; Vesperinas, A. Photoresponsive surfactants in microgel dispersions. Langmuir 2006, 22, 101-105.

(26) Weston, C. E.; Richardson, R. D.; Haycock, P. R.; White, A. J. P.; Fuchter, M. J. Arylazopyrazoles: azoheteroarene photoswitches offering quantitative isomerization and long thermal half-lives. J. Am. Chem. Soc. 2014, 136, 11878-11881.

(27) Stricker, L.; Fritz, E.-C.; Peterlechner, M.; Doltsinis, N. L.; Ravoo, B. J. Arylazopyrazoles as Light-Responsive Molecular Switches in Cyclodextrin-Based Supramolecular Systems. J. Am. Chem. Soc. 2016, 138, 4547-4554.

(28) Moratz, J.; Stricker, L.; Engel, S.; Ravoo, B. J. Controlling Complex Stability in Photoresponsive Macromolecular Host-Guest Systems: Toward Reversible Capture of DNA by Cyclodextrin Vesicles. Macromol. Rapid Commun. 2017, 39, 1700256.

(29) Hernández-Baltazar, E.; Gracia-Fadrique, J. Elliptic solution to the Young-Laplace differential equation. J. Colloid Interface Sci. 2005, 287, 213-216.

(30) Berry, J. D.; Neeson, M. J.; Dagastine, R. R.; Chan, D. Y. C.; Tabor, R. F. Measurement of surface and interfacial tension using pendant drop tensiometry. J. Colloid Interface Sci. 2015, 454, 226-237.

(31) Shen, Y. R.; Ostroverkhov, V. Sum-frequency vibrational spectroscopy on water interfaces: polar orientation of water molecules at interfaces. Chem. Rev. 2006, 106, 1140-1154.

(32) Shen, Y. R. Surfaces probed by nonlinear optics. Surf. Sci. 1994, 299-300, 551-562.

(33) Meltzer, C.; Paul, J.; Dietrich, H.; Jäger, C. M.; Clark, T.; Zahn, D.; Braunschweig, B.; Peukert, W. Indentation and self-healing mechanisms of a self-assembled monolayer - a combined experimental and modeling study. J. Am. Chem. Soc. 2014, 136, 10718-10727.

(34) Ohno, P. E.; Saslow, S. A.; Wang, H.-f.; Geiger, F. M.; Eisenthal, K. B. Phase-referenced nonlinear spectroscopy of the $\alpha$-quartz/water interface. Nat. Commun. 2016, 7, 13587.

(35) Gonella, G.; Lütgebaucks, C.; de Beer, A. G. F.; Roke, S. Second Harmonic and Sum-Frequency Generation from Aqueous Interfaces Is Modulated by Interference. J. Phys. Chem. C 2016, 120, 9165-9173.

(36) Ohno, P. E.; Wang, H.-f.; Geiger, F. M. Second-order spectral lineshapes from charged interfaces. Nat. Commun. 2017, 8, 1032.

(37) Boyer, H. C.; Dutcher, C. S. Statistical Thermodynamic Model for Surface Tension of Aqueous Organic Acids with Consideration of Partial Dissociation. J. Phys. Chem. A 2016, 120, 4368-4375.

(38) Badban, S.; Hyde, A. E.; Phan, C. M. Hydrophilicity of Nonanoic Acid and Its Conjugate Base at the Air/Water Interface. ACS Omega 2017, 2, 5565-5573.

(39) Lu, J. R.; Li, Z. X.; Thomas, R. K.; Staples, E. J.; Tucker, I.; Penfold, J. Neutron reflection from a layer of monododecyl hexaethylene glycol adsorbed at the air-liquid interface: the configuration of the ethylene glycol chain. J. Phys. Chem. 1993, 97, $8012-8020$

(40) Streubel, S.; Schulze-Zachau, F.; Weißenborn, E.; Braunschweig, B. Ion Pairing and Adsorption of Azo Dye/C16TAB Surfactants at the Air-Water Interface. J. Phys. Chem. C 2017, 121, 27992.

(41) Stubenrauch, C.; Miller, R. Stability of foam films and surface rheology: An oscillating bubble study at low frequencies. J. Phys. Chem. B 2004, 108, 6412-6421.

(42) Langevin, D. Aqueous foams: A field of investigation at the frontier between chemistry and physics. ChemPhysChem 2008, 9, 510522.

(43) Blanc, B.; Bonhomme, O.; Brevet, P.-F.; Benichou, E.; Ybert, C.; Biance, A.-L. Electroosmosis near surfactant laden liquid-air interfaces. Soft Matter 2018, 14, 2604.

(44) Kanicky, J. R.; Poniatowski, A. F.; Mehta, N. R.; Shah, D. O. Cooperativity among Molecules at Interfaces in Relation to Various Technological Processes: Effect of Chain Length on the pKa of Fatty Acid Salt Solutions. Langmuir 2000, 16, 172-177.

(45) Schellmann, K.; Preisig, N.; Claesson, P.; Stubenrauch, C. Effects of protonation on foaming properties of dodecyldimethylamine oxide solutions: A pH-study. Soft Matter 2015, 11, 561-571. 